\title{
Quantitative proteomics suggests metabolic reprogramming during ETHE1 deficiency
}

Sahebekhtiari, Navid; Thomsen, Michelle M.; Sloth, Jens Jørgen; Stenbroen, Vibeke; Zeviani, Massimo; Gregersen, Niels; Viscomi, Carlo; Palmfeldt, Johan

\section{Published in:}

Proteomics

Link to article, DOI:

10.1002/pmic.201500336

Publication date:

2016

Document Version

Peer reviewed version

Link back to DTU Orbit

\section{Citation (APA):}

Sahebekhtiari, N., Thomsen, M. M., Sloth, J. J., Stenbroen, V., Zeviani, M., Gregersen, N., Viscomi, C., \& Palmfeldt, J. (2016). Quantitative proteomics suggests metabolic reprogramming during ETHE1 deficiency. Proteomics, 16(7), 1667-1176. https://doi.org/10.1002/pmic.201500336

\section{General rights}

Copyright and moral rights for the publications made accessible in the public portal are retained by the authors and/or other copyright owners and it is a condition of accessing publications that users recognise and abide by the legal requirements associated with these rights.

- Users may download and print one copy of any publication from the public portal for the purpose of private study or research.

- You may not further distribute the material or use it for any profit-making activity or commercial gain

- You may freely distribute the URL identifying the publication in the public portal 


\section{Quantitative Proteomics Suggests Metabolic Reprogramming during}

\section{ETHE1 Deficiency}

Authors: Navid Sahebekhtiari ${ }^{1}$, Michelle M Thomsen ${ }^{1}$, Jens J. Sloth ${ }^{2}$, Vibeke Stenbroen ${ }^{1}$, Massimo Zeviani ${ }^{3}$, Niels Gregersen ${ }^{1}$, Carlo Viscomi ${ }^{3,4}$ and Johan Palmfeldt ${ }^{1 *}$

\section{Affiliations}

1. Research Unit for Molecular Medicine, Department of Clinical Medicine, Aarhus

University and Aarhus University Hospital, Palle Juul-Jensens Boulevard 99, Denmark

2. Research Group for NanoBio Science, National Food Institute, Technical University of

10 Denmark, Mørkhøj Bygade 19, DK-2860 Søborg, Denmark

11 3. Mitochondrial Biology Unit, Medical Research Council, Wellcome Trust/MRC Building, Hills Road, Cambridge CB2 0XY, United Kingdom

4. IRCCS Foundation Neurological Institute "C. Besta", via Temolo,4, 20133 Milano, Italy

*Corresponding Author: email: johan.palmfeldt@clin.au.dk

Received: 21-Aug-2015; Revised: 22-Jan-2016; Accepted: 08-Feb-2016

This article has been accepted for publication and undergone full peer review but has not been through the copyediting, typesetting, pagination and proofreading process, which may lead to differences between this version and the Version of Record. Please cite this article as doi: 10.1002/pmic.201500336.

This article is protected by copyright. All rights reserved. 


\section{Abstract}

3 Deficiency of mitochondrial sulfur dioxygenase (ETHE1) causes the severe metabolic disorder ethylmalonic encephalopathy, which is characterized by early-onset encephalopathy and defective cytochrome $\mathrm{C}$ oxidase because of hydrogen sulfide accumulation. Although the severe systemic consequences of the disorder are becoming clear, the molecular effects are not well defined. Therefore, for further elucidating the effects of ETHE1-deficiency, we performed a large scale quantitative proteomics study on liver tissue from ETHE1-deficient mice. Our results demonstrated a clear link between ETHE1-deficiency and redox active proteins, as reflected by down-regulation of several proteins related to oxidation-reduction, such as different dehydrogenases and cytochrome P450 (CYP450) members. Furthermore, the protein data indicated impact of the ETHE1-deficiency on metabolic reprogramming through up-regulation of glycolytic enzymes and by altering several heterogeneous ribonucleoproteins (hnRNPs), indicating novel link between ETHE1 and gene expression regulation. We also found increase in total protein acetylation level, pointing out the link between ETHE1 and acetylation, which is likely controlled by both redox state and cellular metabolites. These findings are relevant for understanding the complexity of the disease and may shed light on important functions influenced by ETHE1 deficiency and by the concomitant increase in the gaseous mediator hydrogen sulfide.

21 Key words: ethylmalonic encephalopathy, CYP 450, heterogeneous ribonucleoprotein

22 (hnRNP), acetylation, sulfide, Heat shock protein 90 (HSP90), metal, magnesium, iron, zinc, ICP-MS 


\section{Statement of Significance of the Study}

The protein ETHE1 is known to have persulfide dioxygenase activity, and it's homozygous or compound heterozygous deficiency causes the severe ethylmalonic encephalopathy. ETHE1 is also known to be a regulator of the signaling gas sulfide. However, the functional context of ETHE1 is still sparsely understood and we here apply large scale proteomics to show the influence of ETHE1 on several pathways not previously described. Whereas the phenotype during ETHE1 deficiency mainly is neurodevelopmental, the liver proteomics data of this study capture metabolic reprogramming towards glycolysis with concomitant overall increase in protein acetylation. Pronounced decreases of the levels of oxido-reductases indicated altered redox regulation. This together with alterations of several heterogeneous ribonucleoproteins (hnRNPs), important for gene regulation, indicated that ETHE1 has wider

13 impact on the cellular and biochemical constitution than previously thought. 
2 Deficiency of mitochondrial protein ETHE1 causes ethylmalonic encephalopathy (EE) - a severe, inherited metabolic disorder- characterized by encephalopathy, chronic diarrhea and lactic acidosis. ETHE1 has persulfide dioxygenase activity, and when deficient it causes accumulation of hydrogen sulfide $\left(\mathrm{H}_{2} \mathrm{~S}\right)$, which can result in a multitude of biochemical/biological effects $[1,2]$, making ETHE1 biology a challenging topic to study. Two well-known characteristics of ETHE1 deficiency, linked to the toxic levels of sulfide, are inhibition of cytochrome c oxidase (COX) of mitochondrial respiration [3, 4], and shortchain acyl CoA dehydrogenase (SCAD) in fatty acid oxidation pathway [1]. The latter leads to butyryl-CoA accumulation, which after conversion is excreted as ethylmalonic acid (EMA) [5]. The biochemical profile of patients with the EE disorder is also characterized by high lactate, and C4- and C5-acylcarnitines in blood [1]. ETHE1 belongs to the metallo- $\beta$ lactamase superfamily [6] and has sequence homology to glyoxylase II, but unlike glyoxylase II it has a mononuclear iron in the active site [7]. Sulfide is a gasotransmitter [8] with several biological functions, for example vasorelaxation [9], although at high concentration it is toxic to cells [10]. This can explain the clinical findings of vascular symptoms in EE patients, such as petechiae and acrocyanosis. Sulfide is produced endogenously by cystathionine $\beta$-synthase (CBS) and cystathionine $\gamma$-lyase (CSE) by using L-cysteine as a substrate $[11,12]$. ETHE1 takes part in the main pathway for sulfide catabolism, which resides in the mitochondrial matrix and consists of an oxidation reaction catalyzed mainly by sulfide quinone reductase (SQR) and ETHE1 [13, 14]. ETHE1 is a sulfur dioxygenase oxidizing persulfide (e.g. glutathione persulfide) to sulfite $\left(\mathrm{SO}_{3}{ }^{2-}\right)$ while consuming molecular oxygen and water [14, 15]. It has been shown that $\mathrm{O}_{2}$ and sulfide have low degree of coexistence in tissues [16]. Loss of ETHE1 function causes sulfide accumulation and thus hypoxic condition, and as a 
1 vicious cycle hypoxia can raise sulfide levels as oxygen dependent catabolism of sulfide

2 slows down. This close interaction implies the role of sulfide in oxygen sensing [17].

Sulfide is a known redox regulator [15], and our research group have previously described that oxidoreductases show altered levels in fibroblast cells from EE patients [18]. The clinical symptoms of EE and the reported effects of ETHE1 deficiency on mitochondrial fatty acid $\beta$ oxidation, cytoskeleton and branched chain amino acid catabolism [19], exemplify that the ETHE1 deficiency affects several different pathways within the cell. Still further elucidation is necessary to elucidate the interplay between these different molecular mechanisms. These severe systematic consequences of the disease encouraged us to perform large scale proteomics studies on liver tissue from ETHE1-deficient and control mice. Our data confirm the link between ETHE1-deficiency and redox related proteins [18], based on lowered level of several dehydrogenases and of several members of cytochrome P450 (cyp450) family. Metabolic reprogramming towards glycolysis was seen, and a novel link between ETHE1 deficiency and heterogeneous ribonucleoproteins (hnRNPs) was established. 
2 Experimental design

Liver samples from three replicates of both $E t h e 1^{-/-}$and $E t h e I^{+/+}$mice were subjected to proteomics as described previously [20] and below. Due to the smaller size of the Ethe $1^{-/-}$ mice, two livers from these were pooled to get each replicate. Two studies of iTRAQ based comparative proteomics were performed separately on soluble and membrane proteins, respectively, and the data from the two studies were merged prior to pathway analyses and biological interpretations.

\section{ETHE1 deficient mice}

Heterozygous Ethe $1^{-/+}$mice were mated to obtain homozygous Ethe $1^{-/-}$mice. For details on how the Ethel $1^{-/+}$mice were generated see Tiranti et al [1]. The mouse colony was maintained on a129Sv-C57BL/6 mixed background. The liver samples from six Ethe $1^{-/-}$ mice and three $\mathrm{Ethe}^{+/+}$healthy controls, were taken for proteomics studies. Animal studies were approved by the Ethics Committee of the Foundation 'Carlo Besta' Neurological Institute, in accordance with guidelines of the Italian Law D.L. 116/1992 and the EU directive 63-2010.

\section{Proteome fractionation}

Mouse liver tissue was homogenized with a Potter-Elvehjem in cold $\left(4{ }^{\circ} \mathrm{C}\right)$ lysis buffer $(10$ mmol/l Hepes, $\mathrm{pH} 7.2$, with protease inhibitors (Complete, Roche Diagnostics), sonicated three times for $10 \mathrm{sec}$, and briefly centrifuged $\left(400 \times g, 5 \mathrm{~min}, 4^{\circ} \mathrm{C}\right)$ to remove cell debris. The supernatant from homogenization was ultracentrifuged $\left(120,000 \times g, 60 \mathrm{~min} ., 4^{\circ} \mathrm{C}\right)$ to separate 
1 carbonate buffer to remove contaminant soluble proteins and thereafter ultracentrifuged

2 again. The new membrane pellet was dissolved on ice in approximately $1 \%$ Triton $\mathrm{X}-114$. After 15 min incubation at $30^{\circ} \mathrm{C}$, a bottom phase of Triton-X114 micelles was formed and collected by centrifugation $(1300 \times g, 10 \mathrm{~min})$. The Triton-X114 detergent phase, mostly containing membrane proteins $(\mathrm{M})$, and the $\mathrm{S}$ sample were purified by precipitation and analyzed as previously described [21].

\section{Peptide labeling and fractionation}

Two iTRAQ-studies, on $\mathrm{S}$ and $\mathrm{M}$ samples respectively, were performed and three $E t h e 1^{-/}$ and three $E$ the ${ }^{+/+}$samples were included in each. $100 \mu \mathrm{g}$ peptide sample of each replicate was labeled with a specific iTRAQ reagent in accordance with the manufacturer's instructions (Applied Biosystems).The subsequent analytical steps were performed as previously described [21]. Labeled peptides were mixed and purified on a Strong Cation Exchange (SCX) chromatography column followed by elution with 5\% ammonia and 30\% methanol. Pure peptides were dried and resuspended in denaturing buffer, and separated on an Immobiline Drystrip gel (GE Healthcare, Uppsala, Sweden) using isoelectric focusing on a Multiphor II unit (Pharmacia Biotech AB, Uppsala, Sweden). The $\mathrm{pH}$ range was 3.5-4.5 for S peptides and 3-10 for M peptides. The gel strip was cut into twelve pieces, and the peptides were extracted from the gel in two steps with $0.5 \%$ trifluoracetic acid (TFA) in 5\% acetonitrile (ACN). Peptides were purified on PepClean C-18 Spin Columns (Pierce, Rockford, IL, USA) according to manufacturer's protocol prior to nanoLC-MS/MS analysis.

24 The peptide mixtures were separated by liquid chromatography (EASY nano LC from Proxeon, Odense, Denmark) coupled to mass spectrometry (LTQ-Orbitrap, Thermo Fisher 
1 Scientific, Waltham, USA), through a nano-electrospray source with stainless steel emitter

2 (Proxeon). Each sample was LC-MS analysed twice. The peptides were separated on a reverse phase column, $75 \mu \mathrm{m}$ in diameter and $100 \mathrm{~mm}$ long, packed with $3.5 \mu \mathrm{m}$ Kromasil C18 particles (Eka Chemicals, Bohus, Sweden) at a flow of $300 \mathrm{nl} /$ minute using a 100 minutes gradient of $\mathrm{AcN}$ in $0.4 \%$ acetic acid, starting with $5 \%$ and ending with $35 \% \mathrm{AcN}$. The mass spectrometry detection was full scan $(\mathrm{m} / \mathrm{z} 400-2000)$ with Orbitrap detection at resolution $\mathrm{R}=60,000$ (at $\mathrm{m} / \mathrm{z}$ 400) followed by up to four data-dependent MS/MS scans, with linear ion trap (LTQ) detection of the most intense ions. Dynamic exclusion of $25 \mathrm{sec}$ was employed as well as rejection of charge state +1 . Pulsed Q dissociation (PQD) fragmentation was performed with activation time of $0.1 \mathrm{sec}$, two microscans, normalized collision energy of 33, and activation Q of 0.7 .

\section{Database searches and statistics}

The raw data files were processed using extract_msn.exe (Thermo Fisher Scientific, released $2 / 15 / 2009$ ) to generate peak lists of the tandem spectra. To obtain protein identification and iTRAQ reporter quantification data the MS data were subjected to database searches in Proteome Discoverer 1.3 (Thermo) using the algorithm of Mascot (www.matrixscience.com) version 2.2.04 (Matrix Science, London, UK) for peptide and protein identification. In each study, the twelve different peptide fractions were MS-analysed in duplicate and all generated peak lists were merged. The merged files were searched against the mouse database, Mus musculus SwissProt_2012_3.fasta with 16520 sequences, using the MudPIT scoring algorithm of Mascot. Full scan tolerance was $5 \mathrm{ppm}$ and MS/MS tolerance was 0.5 Da. Setting of trypsin digestion was cleavage at C-terminal of lysine and arginine except before proline, and up to two missed cleavages were accepted. Fixed modifications were those 
1 originating from iTRAQ protocol: iTRAQ-8plex of lysine and N-terminal and methylthio

2 modification of cysteines, whereas oxidation of methionine and iTRAQ-8plex of tyrosine were set as variable modifications. The iTRAQ labeling efficiency was estimated from database searches where the iTRAQ labeling of N-termini and lysine residues, and also lysine acetylation, were set as dynamic modifications. iTRAQ labeling was found to be present on more than $99 \%$ of the expected sites (calculated from more than 50,000 peptide scans), and was therefore subsequently set as a fixed modification. Less than $0.7 \%$ of the lysine residues were carrying acetylation instead of iTRAQ label. Deacetylase inhibitors were not used in sample preparation of the iTRAQ samples since loss of the lysine acetylations can be an advantage during the iTRAQ based quantification of the relative amounts of the proteins. The low degree of acetylation sites indicates negligible impact of the lysine acetylation on the accuracy of the iTRAQ-based protein quantitation in this study. The significance level of protein identifications was set to 0.001 , which resulted in a false discovery rate (FDR) of less than 0.003 when searched in Mascot against the decoy database of random sequences. iTRAQ values were reported for proteins with five or more measured iTRAQ scan values from peptides with expectation values of 0.02 or below. The quantification data of each biological sample were normalized to protein median to compensate for possible variation in starting material. Only proteins detected from at least two peptides were included in the statistical calculations. Ratios for each protein were reported as significantly different from 1.0 if they passed two tests: 1$)$ a fold change criterion (>1.25) based on two times the global standard error and 2) a two-tailed student's $t$-test $(\mathrm{p}<0.05)$ for equal variance data. Proteins detected in both soluble and membrane fraction and with significance in the fraction with lowest abundance (as estimated from number of scans) were checked for coherency in fold change direction in the other fraction, and omitted from further data descriptions if found incoherent. Four such cases were found: Atp5a1, Dbt, Ganab and RPS14. 
1 All the mass spectrometry proteomics data have been deposited to the ProteomeXchange

2 Consortium via the PRIDE partner repository with the dataset identifier PXD002741 and 10.6019/PXD002741 for the membrane proteome fraction (Username: reviewer73951@ebi.ac.uk, Password: 9LhL1Cr8) and dataset identifier PXD002742 and 10.6019/PXD002742 for the supernatant proteome fraction (Username: reviewer00023@ebi.ac.uk, Password: I40pJZR8) [22].

\section{Data analysis}

To analyze the protein lists derived from MS and extracting biological meaning from the lists we applied the DAVID bioinformatic resource and used the 967 proteins from the study as a background. Furthemore, to better understand what functional interactions these siginificantly changed proteins have we used STRING software.

\section{Western blot analysis of protein acetylations}

Western blot analysis was performed onproteins extracted from the liver samples in the presence of a cocktail of deacetylase inhibitors: $10 \mu \mathrm{M}$ trichostatin A (TSA), $10 \mathrm{mM}$ sodium butyrate, $2 \mu \mathrm{M}$ suberoylanilide hydroxamic acid (SAHA, vorinostat) and $10 \mathrm{mM}$ nicotinamide. The primary antibody was anti-acetyllysine antibody (Cell signaling), and the secondary was anti-rabbit-HRP antibody (Dako, Denmark). Detailed procedure can be found in Supplementary Materials and Methods.

\section{Parallel Reaction Monitoring (PRM) MS}

Based on the large-scale study, proteins were selected for validation by targeted MS analysis using Parallel Reaction Monitoring (PRM) on nanoLC coupled to Q-Exactive Plus MS 
1 (Thermo). Protein extracts from livers of six $E t h e 1^{-/-}$and three $E t h e 1^{+/+}$mice were prepared

2 in the same way as Western blot samples, and subsequently proteins were in-gel trypsin digested (Supplementary Materials and Methods). Proteins selected for PRM validation were imported to the software Skyline where tryptic in silico digestions were carried out. More details on the targeted proteins and peptides can be found in Supplementary Materials and Methods.

\section{Elemental analysis by ICP-MS}

The concentrations of elements (iron, zink, magnesium and sulfur) were determined by inductively coupled plasma mass spectrometry (ICP-MS) following micro-scaled pressurised digestion [23] Briefly, mouse liver tissue samples (10-20 mg) were digested in a microwave oven (Multiwave 3000, Anton Paar, Graz, Austria) in $5 \mathrm{ml}$ glass vials using $250 \mu \mathrm{L}$ concentrated nitric acid and $100 \mu \mathrm{L}$ hydrogenperoxide (both Merck, Darmstadt, Germany).

14 The digests were diluted to a volume of $5 \mathrm{~mL}$ with milliQ water and subsequently their 15 content of elements was determined using external calibration by ICP-QQQ-MS (Agilent 16 8800, Agilent Technologies, Yokogawa, Japan) run in the helium $\left({ }^{57} \mathrm{Fe},{ }^{66} \mathrm{Zn},{ }^{24} \mathrm{Mg}\right)$ and oxygen modes $\left({ }^{34} \mathrm{~S}(32->48)\right.$, respectively.

\section{Results and Discussion}


1 To elucidate the impact of deficiency of the persulfide dioxygenase ETHE1, we performed

2 large scale proteomics on liver tissue samples from ETHE1-deficient mice and control mice.

The mass spectrometry based proteomics yielded identification and quantification data for 595 and 524 proteins in soluble (S) and membrane (M) protein fractions, respectively, with 152 proteins detected in both. Detection in both fractions can be due to cross-contamination of abundant proteins (eg albumin), however, the 152 proteins are mostly enzymes and are included in the statistical analysis since many enzymes are known to have partial or temporary membrane association with biological relevance. Table S1 lists the data of all the 967 unique proteins (1119 protein entries). 137 proteins passed the tests of statistical significance $(\mathrm{p}<0.05$ and fold change $>1.25$-fold change) (Table S2). These proteins were subjected to bioinformatics analyses.

\section{Bioinformatic analysis of protein functionalities}

DAVID and STRING software tools were applied to gain functional and biological enrichment of the proteins with significantly altered levels and to elucidate how these proteins are linked to each other, and to identify enriched functional annotation terms within the datasets. All 137 statistically significant proteins were subjected to DAVID with 1119 obtained proteins as background data set to identify enriched functional annotation (Table 1). There were 28 proteins with the functional annotation "Mitochondrion", where ETHE1 is located. The other functional categories cover a variety of functionalities indicating wide biochemical and functional impact of ETHE1 deficiency, as described in sections below. The same data-set was applied to STRING in order to elucidate more details about protein-protein interactions and functional associations (Figure S1A, B).

\section{Hydrogen sulfide and oxidation-reduction}

This article is protected by copyright. All rights reserved. 
1 Among the categories obtained in Table 1 two were particularly pronounced; oxidation-

2 reduction and metal ion binding proteins (Table 2). 12 proteins were common for these two groups, and since all proteins were down-regulated in our study, there seems to be a link between sulfide accumulation and proteins involved in oxidation-reduction. Of the proteins which have metal as a cofactor, six depend on iron for activity including four members of the Cytochrome P450 family, while the rest need zinc or other metals such as magnesium or manganese.

Approximately $44 \%$ of oxidoreductases have been described as metalloenzymes and $81 \%$ of them are iron-dependent [24]. Since $60 \%$ of the Oxidation-Reduction proteins (12 out of the 20) in Table 2 are metal-binding, there might be an interaction between sulfide and metalloproteins, notwithstanding different kind of metals are represented. Furthermore, according to the DAVID analyses, six proteins were solely in the metal binding cluster category (Table 2). To better delineate whether it is the oxidoreductase function or the metal binding protein property being more central we performed elemental analysis of metals.

\section{Elemental analysis of metals and sulfur}

Sulfide is able to precipitate iron and zinc ions at neutral $\mathrm{pH}$ to form metal sulfides [25], which might lead to intracellular accumulation of both the metals and the element sulfur. We therefore quantitated the total, bound and unbound, amount of zinc and iron together with magnesium (also a co-factor in Table 2). The concentrations of zinc, iron and magnesium in liver tissue of ETHE1-deficient and healthy control mice were measured by inductively coupled plasma mass spectrometry (ICP-MS) in fully hydrolysed samples. The concentrations (mean \pm standard deviation) for ETHE1 deficient $(n=6)$ and controls $(n=3)$, respectively, were for zinc $45 \pm 13$ vs $42 \pm 5$, for iron $180 \pm 121$ vs $222 \pm 79$, and for magnesium $350 \pm 16$ vs $372 \pm 36 \mu \mathrm{g} / \mathrm{kg}$. No statistical significant changes were observed between the two 

found to be $3420 \pm 415$ and $3550 \pm 268 \mu \mathrm{g} / \mathrm{kg}$ in ETHE1 deficient and control livers, respectively. Since the concentrations of the measured metals and sulfur were unaltered it indicates that the observed proteomics alterations of the oxidation-reduction proteins instead are caused by the cellular redox status and specific biological regulation.

\section{Cytochrome P450 and oxidative stress}

Of the 20 oxidation-reduction proteins significantly down-regulated in ETHE1-deficient mice, there were four cytochrome P450 (CYP) proteins. Cytochrome P450 is an endoplasmic reticulum-anchored family of heme proteins [26] that are important for various kinds of detoxifications reactions. CYPs can produce superoxide anion and hydrogen peroxide [27], which in turn may release 'catalytic' iron and generate potent oxidants. Four members of the large CYP family showed clearly lowered levels in the ETHE1-deficient mice: CYP1A2, CYP2C29, CYP2C70 and CYP2D10 (Figure 1A), and CYPs of these CYP1, CYP2 and CYP4 families typically have activities in eicosanoid metabolism [28]. CYP1A2 has previously been shown to be down-regulated under acute sulfide poisoning [29]. It has also been shown that CYP450 inhibition decreases hydrogen sulfide induced oxidative stress [30]. Thus the observed down-regulation of four CYP proteins may be a way for the cells to alleviate sulfide-induced stress.

Four proteins with relation to oxidative stress or glutathione were significantly downregulated (Figure 1B); catalase, glutathione s transferase 2 and 3 (GSTM2, GSTM3), and methionine sulfoxide reductase (MSR). Superoxide dismutase (SOD2) was previously found down-regulated in mitochondria from ETHE1 deficient human fibroblasts [18], but was not 
1 detected in our study showed a general tendency to be down-regulated (13 out of 18 were

2 below 1.0, with a median ratio of 0.8 ) (Figure 1B). These observations together, indicate that redox balance and antioxidant activity were affected in ETHE1-deficient mice.

\section{Metabolic reprogramming in ETHE1 deficiency}

Our data clarified a clear link between ETHE1 and energy status of cell, through the significant up-regulation of several metabolic pathways (Figure 1C and1D), which likely can compensate for the lack of energy caused by the established dysfunction of mitochondrial metabolism, including lactic acidosis and SCAD inhibition. Two important proteins in glycolysis were found to have increased levels: fructose bisphosphate aldolase (FBA) and pyruvate kinase (PK). FBA catalyzes the reversible reaction of Fructose 1,6 bisphosphate to Glyceraldehyde 3-phosphate in glycolysis. PK is the last enzyme in glycolysis, which is responsible for producing pyruvate from phosphoenolpyruvate [31]. Lactate dehydrogenase using pyruvate as substrate was also up-regulated. Apart from lactate dehydrogenase and the glycolytic FBA and PK with significant increases, six other enzymes of glycolysis were successfully quantitated, however not found significant and with levels ranging from 0.82 to 1.17 (Figure S2). Increased levels of glycolytic enzymes (FBA and PK) in combination with increased LDH can lead to increased production of lactate, which is in line with the fact that patients with mutation in ETHE1 show lactic acidosis [32].

Pyruvate carboxylase, which is responsible for pyruvate carboxylation and converting it to oxaloacetate, was up-regulated in our study [33]. Oxaloacetate is an important intermediate in metabolism, linking carbohydrate, lipid, amino acid and nucleotide metabolism [34], and can either enter Krebs cycle or gluconeogenesis pathway. Another sign of the shift from mitochondrial respiration towards glycolysis was calcium-binding mitochondrial carrier 
1 protein (citrin) up-regulation. Citrin is the hepatic isoform of the mitochondrial aspartate-

2 glutamate carrier [35], and is known to participate in gluconeogenesis [36], which keeps glycolysis reactions active. Up-regulation of citrin in our study further support the notion that ETHE1-deficient cells favored glycolysis rather than mitochondrial respiration, probably driven by compromised COX and thus respiratory chain.

Enzymes of fatty acid oxidation (FAO) and branched chain amino acid (BCAA) metabolism were found up-regulated in ETHE1-deficient mice, namely medium-chain acyl-CoA dehydrogenase (ACADM), very-long-acyl-CoA dehydrogenase (ACADVL), enoyl-CoA hydratase, propionyl-CoA carboxylase, and dihydrolipoyl dehydrogenase (DLD).

10

\section{ETHE1 deficiency and gene expression via hnRNP}

The heterogeneous nuclear ribonucleoprotein (hnRNP) contains RNA-binding proteins which associate with heterogeneous nuclear RNAs and are responsible for regulating gene expression in response to stresses such as hypoxia [37]. In our study six different hnRNP were found to have elevated level in the ETHE1-deficient mouse liver (Figure 1E), which is consistent with data describing link between redox homeostasis and hnRNP proteins [38]. As discussed above glycolysis is favored during ETHE1-deficiency, indicating metabolic reprogramming, known as the Warburg effect [39]. It has been shown that pyruvate kinases are regulated by heterogeneous nuclear ribonucleoprotein (hnRNP), and that the isoform PKM2 is increased by hnRNPA1 and hnRNPA2 [40]. Interestingly, our data showed increased level of pyruvate kinase and hnRNP proteins (eg. hnRNPA1), which indicate a regulatory function of hnRNPs in cells with mitochondrial dysfunctions due to ETHE1 deficiency. Furthermore, hnRNP A1 has splicing activity on the ACADM (MCAD) altered in 
1 this study [41].Recently hnRNP A1 was found to be over-expressed in cancer [42], and

2 Warburg effect is present in cancer and also in inborn errors of metabolism (IEM) [43]. The differentially altered hnRNPs in our study contain 6 to 18 acetylated lysine residues [44], which suggests that acetylation has impact on hnRNP regulated splicing and gene regulation. Acetylation has recently been described to increase stability of hnRNPs, e.g. hnRNP A1, I and L [45]. A growing amount of data obtained through different approaches indicates the importance of splicing activity of hnRNPs in response to metabolic state of the cells, including protein acetylation status.

\section{Protein acetylation}

10 Our results showed a clear link between ETHE1 and altered energy metabolism, which in turn is known to be influenced by the post-translational modification (PTM), such as protein acetylation [46]. Based on recent proteomics studies, a majority of acetylation events occur in the mitochondrial proteome and seems to be the most prevalent mitochondrial PTM; regulating signaling networks through metabolic intermediates in glycolysis, Krebs cycle and fatty acid $\beta$-oxidation $[47,48]$. We decided to analyse the total level of protein acetylation, in order to investigate how it was affected in ETHE1-deficient mice compared to healthy mice. The level of acetylated proteins was visualized by western blotting, using an antibody against acetylated lysine residues (Figure 2). Total protein staining with Sypro Ruby was used to verify equal loading (Figure S3). Total protein acetylation pattern exhibited increased intensities in ETHE1-deficient mice ( $p$-value: 0.07), and since a high number of bands had increased intensities it indicates a broad cellular alteration. Histones are well-characterized highly acetylated small proteins [49]. Interestingly, we did not detect clear changes in 14-16 $\mathrm{kDa}$ area where histones normally migrate as dominating proteins (Figure 2). Acetylation is catalyzed by lysine acetyltransferases (KATs), using acetyl-CoA as a cofactor, and can be 
1 removed by lysine deacetylases (KDACs), consisting of a large family of histone

2 deacetylases (HDAC) and Sirtuins [50]. Besides enzymatic reaction, acetylation occurs nonenzymatically, via direct contact of proteins with acetyl-CoA. Several non-acetyl metabolites such as $\mathrm{NAD}^{+}$have been shown to be key regulators of deacetylases. The NAD ${ }^{+} / \mathrm{NADH}$ ratio, which reflects the redox state of the cell, regulates the activities of the sirtuins, and thus influences global acetylation levels [51]. The glycolysis product pyruvate is known as inhibitor of several histone deacetylases such as HDAC1 and HDAC3 [52]. So, shifting metabolism from mitochondria toward glycolysis in ETHE1-deficient mice could be correlated with the generally increased protein acetylation. Butyrate is also known as HDAC inhibitor metabolite [53], and SCAD inhibition in ETHE1 deficiency leads to butyrate accumulation that in turns can have inhibitory impact on HDACs. These findings point to a link between ETHE1 and acetylation, where both redox state and cellular metabolites can influence acetylation and deacetylation activity.

\section{Hydrogen sulfide and HSP90}

HSP90 is an abundant stress protein, mostly located in the cytoplasm, playing an important role in various cellular processes such as protein folding, protein degradation and signal transduction cascades [54]. In ETHE1-deficient mice HSP90 was down-regulated (Figure 1F). It has previously been shown that HSP90 interacts with TPR domain proteins (tetratricopeptide repeat), such as proteins of the CYP family [55], and our study showed simultaneous down-regulation of both. Since HSP90 has been shown to be up-regulated in oxidative stress $[54,56]$, down-regulation of HSP90 in our study suggests a reductive, instead of an oxidative, condition during ETHE1 deficiency. 
1 Cytoskeleton proteins and Epithelial-Mesenchymal Transition (EMT)

2 Vimentin, a major constituent of the Intermediate Filament (IF) family of proteins was found up-regulated. Vimentin is central for maintaining cellular integrity in eg mesenchymal cells [57] and is a marker for Epithelial-Mesenchymal Transition (EMT) which is an important mechanism contributing to cancer $[58,59]$. Our results show that besides vimentin, other cytoskeleton proteins were also affected by ETHE1 deficiency (Figure 1G).

\section{Comparison with previous studies}

Our results on cytoskeletal proteins are in line with elevated levels of cytoskeletal proteins previously found in muscle and brain tissues of ETHE1-deficient mice [19]. Furthermore, Hildebrandt et al also found a bioinformatic overrepresentation of acetylated proteins, and 12 here we experimentally described the presence of increased protein acetylation. They performed 2D-PAGE based proteomics and identified 16 differentially altered proteins which

14 we also detected, and out of those we could also get clear significance for 6 proteins (ATP5A1, ECHS1, FKPB4, PRDX1, RGN and SUOX), despite the very different procedures of the two studies.

\section{Validation by targeted MS protein analysis}

19 Accurate and precise quantification of proteins can be done by targeted proteomics via selected reaction monitoring (SRM), or the similar Parallel Reaction monitoring (PRM) on high mass accuracy instruments [60]. We applied PRM on unfractionated samples with addition of heavy labeled peptide internal standards. 
1 A group of peptides were selected to measure VLCAD, HSP90, APOE and CYP1A2, chosen

2 as four representative proteins significant in the iTRAQ study (Table 3). All but APOE supported the data from the main study. VLCAD level was 1.45 fold up-regulated, and HSP90 and CYP1A2 were down-regulated 0.66 fold and 0.45 fold respectively. VLCAD, HSP90 and CYP1A2 are examples of three proteins clearly regulated during the ETHE1 deficient condition and they illustrate the wide impact the ETHE1 can have on the cell pathophysiology.

\section{Mitochondrial proteins}

ETHE1 is localized to mitochondria and elicit effects there, eg the negative effects of ETHE1 deficiency on mitochondrial cytochrome C oxidase. Several mitochondrial proteins had elevated levels, eg citrin, and enzymes of fatty acid oxidation and branched chain amino acid metabolism. To test whether these proteins were specifically altered or it was due to a general increase in mitochondrial amounts five reference proteins, chosen to represent stably expressed proteins of both mitochondria (VDAC, HSP60) and cytosol (PGM, RL7A, and RL19), were assayed by PRM. The reference proteins were detected in all samples and at a rather constant level. By calculating the ratio of VDAC and HSP60 versus the PGM, RL7A and RL19 an estimate of mitochondrial content was obtained which showed close to same level in ETHE1 deficient and control samples (ratio: 0.93), indicating that the group of mitochondrial proteins found significant were specifically altered. The level of SCAD and ECHD1 proteins were assayed since the first is known to be inhibited by ETHE1 deficiency and the second can catalyze the reaction ethylmanoyl-CoA to butanoyl-CoA, with relation EMA [61]. None of them were found to have altered level in line with the data of the main iTRAQ study. 


\section{Concluding remarks:}

In conclusion, by performing large scale quantitative proteomics on ETHE1-deficient mice liver tissues a clear link between ETHE1-deficiency and cellular redox state was observed, since several oxidoreductases and dehydrogenases showed decreased levels, similar to what has been shown on cultivated fibroblasts [18]. In addition to confirming previous results on ETHE1 impact on BCAA and FA catabolism [19], we report metabolic reprogramming and shifting metabolism toward glycolysis. The current data also demonstrate increased protein acetylation and novel links between ETHE1 and gene expression through hnRNPs.

\section{References}

1.

Tiranti, V., C. Viscomi, T. Hildebrandt, I. Di Meo, et al., Loss of ETHE1, a mitochondrial dioxygenase, causes fatal sulfide toxicity in ethylmalonic encephalopathy. Nat Med, 2009. 15(2): p. 200-205. 
12.

Burlina, A.B., C. Dionisivici, M.J. Bennett, K.M. Gibson, et al., A New Syndrome with

2 Ethylmalonic Aciduria and Normal Fatty-Acid Oxidation in Fibroblasts. J Pediatr, 1994. 124(1): p. 79-

86.

3. Cooper, C.E. and G.C. Brown, The inhibition of mitochondrial cytochrome oxidase by the gases carbon monoxide, nitric oxide, hydrogen cyanide and hydrogen sulfide: chemical mechanism and physiological significance. J Bioenerg Biomembr, 2008. 40(5): p. 533-539.

4.

Di Meo, I., G. Fagiolari, A. Prelle, C. Viscomi, et al., Chronic Exposure to Sulfide Causes Accelerated Degradation of Cytochrome c Oxidase in Ethylmalonic Encephalopathy. Antioxid Redox Sign, 2011. 15(2): p. 353-362.

5. Gregersen, N., B.S. Andresen, and P. Bross, Prevalent mutations in fatty acid oxidation 11 disorders: diagnostic considerations. Eur J Pediatr, 2000. 159: p. S213-S218.

6. Daiyasu, H., K. Osaka, Y. Ishino, and H. Toh, Expansion of the zinc metallo-hydrolase family of the beta-lactamase fold. Febs Lett, 2001. 503(1): p. 1-6.

7.

McCoy, J.G., C.A. Bingman, E. Bitto, M.M. Holdorf, et al., Structure of an ETHE1-like protein from Arabidopsis thaliana. Acta Crystallogr D, 2006. 62: p. 964-970.

8.

Gadalla, M.M. and S.H. Snyder, Hydrogen sulfide as a gasotransmitter. J Neurochem, 2010. 113(1): p. 14-26.

9. Yang, G.D., L.Y. Wu, B. Jiang, W. Yang, et al., H(2)S as a physiologic vasorelaxant: Hypertension in mice with deletion of cystathionine gamma-lyase. Science, 2008. 322(5901): p. 587590.

10.

Kabil, O. and R. Banerjee, Characterization of Patient Mutations in Human Persulfide Dioxygenase (ETHE1) Involved in H2S Catabolism. Journal of Biological Chemistry, 2012. 287(53): p. 44561-44567. endogenous gaseous K-ATP channel opener. Embo J, 2001. 20(21): p. 6008-6016. 
112. Kabil, O., V. Vitvitsky, P. Xie, and R. Banerjee, The Quantitative Significance of the Transsulfuration Enzymes for H2S Production in Murine Tissues. Antioxid Redox Sign, 2011. 15(2): p. 363-372.

13. Jackson, M.R., S.L. Melideo, and M.S. Jorns, Human Sulfide:Quinone Oxidoreductase Catalyzes the First Step in Hydrogen Sulfide Metabolism and Produces a Sulfane Sulfur Metabolite. Biochemistry-Us, 2012. 51(34): p. 6804-6815.

14. Hildebrandt, T.M. and M.K. Grieshaber, Three enzymatic activities catalyze the oxidation of sulfide to thiosulfate in mammalian and invertebrate mitochondria. Febs J, 2008. 275(13): p. 3352-3361.

15.

Kabil, O., N. Motl, and R. Banerjee, $\mathrm{H} 2 \mathrm{~S}$ and its role in redox signaling. Bba-Proteins

11 Proteom, 2014. 1844(8): p. 1355-1366.

16.

Doeller, J.E., T.S. Isbell, G. Benavides, J. Koenitzer, et al., Polarographic measurement of hydrogen sulfide production and consumption by mammalian tissues. Anal Biochem, 2005. 341(1): p. 40-51.

17.

Olson, K.R., R.A. Dombkowski, M.J. Russell, M.M. Doellman, et al., Hydrogen sulfide as an oxygen sensor/transducer in vertebrate hypoxic vasoconstriction and hypoxic vasodilation. J Exp Biol, 2006. 209(20): p. 4011-4023.

18.

Palmfeldt, J., S. Vang, V. Stenbroen, E. Pavlou, et al., Proteomics Reveals that Redox Regulation Is Disrupted in Patients with Ethylmalonic Encephalopathy. J Proteome Res, 2011. 10(5): p. $2389-2396$.

19.

Hildebrandt, T.M., I. Di Meo, M. Zeviani, C. Viscomi, et al., Proteome adaptations in Ethe1-deficient mice indicate a role in lipid catabolism and cytoskeleton organization via posttranslational protein modifications. Bioscience Rep, 2013. 33: p. 575-584. 
120.

Henningsen, K., J. Palmfeldt, S. Christiansen, I. Baiges, et al., Candidate Hippocampal

Biomarkers of Susceptibility and Resilience to Stress in a Rat Model of Depression. Molecular \& Cellular Proteomics, 2012. 11(7).

21.

Baiges, I., J. Palmfeldt, C. Blade, N. Gregersen, et al., Lipogenesis is decreased by grape seed proanthocyanidins according to liver proteomics of rats fed a high fat diet. Mol Cell Proteomics, 2010. 9(7): p. 1499-513.

22.

Vizcaino, J.A., R. Cote, F. Reisinger, H. Barsnes, et al., The Proteomics Identifications database: 2010 update. Nucleic Acids Res, 2010. 38(Database issue): p. D736-42.

23.

Hansen, T.H., K.H. Laursen, D.P. Persson, P. Pedas, et al., Micro-scaled highthroughput digestion of plant tissue samples for multi-elemental analysis. Plant Methods, 2009. 5.

24. Andreini, C., I. Bertini, G. Cavallaro, G.L. Holliday, et al., Metal ions in biological catalysis: from enzyme databases to general principles. J Biol Inorg Chem, 2008. 13(8): p. 1205-1218.

25.

Wiberg, E., N. Wiberg, and A.F. Holleman, Inorganic chemistry. 2001, Academic Press, :San Diego. p. xxxix, 1884 p.

26.

Gonzalez, F.J., The Molecular-Biology of Cytochrome P450s. Pharmacol Rev, 1988. 40(4): p. 243-288.

27.

Karuzina, I.I. and A.I. Archakov, The Oxidative Inactivation of Cytochrome-P450 in Monooxygenase Reactions. Free Radical Bio Med, 1994. 16(1): p. 73-97.

28.

Nebert, D.W., K. Wikvall, and W.L. Miller, Human cytochromes P450 in health and disease. Philosophical transactions of the Royal Society of London. Series B, Biological sciences, 2013. 368(1612): p. 20120431.

29.

Wang, X.Q., M.C. Chen, X.X. Chen, J.S. Ma, et al., The Effects of Acute Hydrogen Sulfide Poisoning on Cytochrome P450 Isoforms Activity in Rats. Biomed Res Int, 2014. 
130.

Eghbal, M.A., P.S. Pennefather, and P.J. O'Brien, H2S cytotoxicity mechanism involves

reactive oxygen species formation and mitochondrial depolarisation. Toxicology, 2004. 203(1-3): p. 69-76.

31.

Lehninger, A.L., D.L. Nelson, and M.M. Cox, Lehninger principles of biochemistry. 6th ed. 2013, New York: W.H. Freeman.

32.

Tiranti, V., P. D'Adamo, E. Briem, G. Ferrari, et al., Ethylmalonic encephalopathy is caused by mutations in ETHE1, a gene encoding a mitochondrial matrix protein. Am J Hum Genet, 2004. 74(2): p. 239-252.

33.

Wexler, I.D., Y.F. Du, M.V. Lisgaris, S.K. Mandal, et al., Primary Amino-Acid-Sequence and Structure of Human Pyruvate-Carboxylase. Bba-Mol Basis Dis, 1994. 1227(1-2): p. 46-52.

34.

Wallace, J.C., My Favorite Pyruvate Carboxylase Feature. lubmb Life, 2010. 62(7): p. 535-538.

35.

Palmieri, L., B. Pardo, F.M. Lasorsa, A. del Arco, et al., Citrin and aralar1 are Ca2+stimulated aspartate/glutamate transporters in mitochondria. Embo J, 2001. 20(18): p. 5060-5069.

36.

Krebs, H.A., T. Gascoyne, and B.M. Notton, Generation of Extramitochondrial

Reducing Power in Gluconeogenesis. Biochem J, 1967. 102(1): p. 275-\&.

1737

Zhou, Z.J., Z. Dai, S.L. Zhou, X.T. Fu, et al., Overexpression of HnRNP A1 promotes tumor invasion through regulating CD44v6 and indicates poor prognosis for hepatocellular carcinoma. Int J Cancer, 2013. 132(5): p. 1080-1089.

38.

Hayakawa, H., A. Fujikane, R. Ito, M. Matsumoto, et al., Human proteins that specifically bind to 8-oxoguanine-containing RNA and their responses to oxidative stress. Biochem Bioph Res Co, 2010. 403(2): p. 220-224.

Warburg, O., Origin of Cancer Cells. Science, 1956. 123(3191): p. 309-314. deregulate pyruvate kinase mRNA splicing in cancer. Nature, 2010. 463(7279): p. 364-U114. 

Inhibited by Binding of hnRNP A1 to a Common ESS Motif That Spans the 3 ' Splice Site. Hum Mutat, 2011. 32(2): p. 220-230.

42. Pino, I., R. Pio, G. Toledo, N. Zabalegui, et al., Altered patterns of expression of members of the heterogeneous nuclear ribonucleoprotein (hnRNP) family in lung cancer. Lung Cancer-J Iaslc, 2003. 41(2): p. 131-143.

43.

Olsen, R.K., N. Cornelius, and N. Gregersen, Redox signalling and mitochondrial stress responses; lessons from inborn errors of metabolism. J Inherit Metab Dis, 2015.

44.

Hornbeck, P.V., J.M. Kornhauser, S. Tkachev, B. Zhang, et al., PhosphoSitePlus: a comprehensive resource for investigating the structure and function of experimentally determined post-translational modifications in man and mouse. Nucleic Acids Res, 2012. 40(D1): p. D261-D270. 45. Koumbadinga, G.A., N. Mahmood, L. Lei, Y.C. Kan, et al., Increased stability of heterogeneous ribonucleoproteins by a deacetylase inhibitor. Bba-Gene Regul Mech, 2015. 1849(8): p. 1095-1103.

46.

Zhao, S.M., W. Xu, W.Q. Jiang, W. Yu, et al., Regulation of Cellular Metabolism by Protein Lysine Acetylation. Science, 2010. 327(5968): p. 1000-1004. Protein Complexes and Co-Regulates Major Cellular Functions. Science, 2009. 325(5942): p. 834-840. 48. Kim, S.C., R. Sprung, Y. Chen, Y.D. Xu, et al., Substrate and functional diversity of lysine acetylation revealed by a proteomics survey. Mol Cell, 2006. 23(4): p. 607-618.

49.

Tessarz, P. and T. Kouzarides, Histone core modifications regulating nucleosome structure and dynamics. Nat Rev Mol Cell Bio, 2014. 15(11): p. 703-708. Iysine acetylation links metabolism and cell signalling. Nat Rev Mol Cell Bio, 2014. 15(8): p. 536-550. 
$151 . \quad$ Rardin, M.J., J.C. Newman, J.M. Held, M.P. Cusack, et al., Label-free quantitative

2 proteomics of the lysine acetylome in mitochondria identifies substrates of SIRT3 in metabolic pathways. P Natl Acad Sci USA, 2013. 110(16): p. 6601-6606.

52.

Thangaraju, M., K.N. Carswell, P.D. Prasad, and V. Ganapathy, Colon cancer cells maintain low levels of pyruvate to avoid cell death caused by inhibition of HDAC1/HDAC3. Biochem J, 2009. 417: p. 379-389.

53.

Donohoe, D.R., L.B. Collins, A. Wali, R. Bigler, et al., The Warburg Effect Dictates the Mechanism of Butyrate-Mediated Histone Acetylation and Cell Proliferation. Mol Cell, 2012. 48(4): p. 612-626.

54.

Csermely, P., T. Schnaider, C. Soti, Z. Prohaszka, et al., The 90-kDa molecular

11 chaperone family: Structure, function, and clinical applications. A comprehensive review. Pharmacol Therapeut, 1998. 79(2): p. 129-168.

55. Periyasamy, S., T. Hinds, L. Shemshedini, W. Shou, et al., FKBP51 and Cyp40 are positive regulators of androgen-dependent prostate cancer cell growth and the targets of FK506 and cyclosporin A. Oncogene, 2010. 29(11): p. 1691-1701.

56. Cabreiro, F., C.R. Picot, M. Perichon, J. Mary, et al., Identification of proteins 17 undergoing expression level modifications in WI-38SV40 fibroblasts overexpressing methionine sulfoxide reductase A. Biochimie, 2007. 89(11): p. 1388-1395.

57. Satelli, A. and S.L. Li, Vimentin in cancer and its potential as a molecular target for cancer therapy. Cell Mol Life Sci, 2011. 68(18): p. 3033-3046.

58. Soini, Y., H. Tuhkanen, R. Sironen, I. Virtanen, et al., Transcription factors zeb1, twist and snai1 in breast carcinoma. Bmc Cancer, 2011. 11. 59. Li, L.P., C.H. Lu, Z.P. Chen, F. Ge, et al., Subcellular proteomics revealed the epithelial- 
160.

Calvo, E., E. Camafeita, B. Fernandez-Gutierrez, and J.A. Lopez, Applying selected

reaction monitoring to targeted proteomics. Expert Rev Proteomic, 2011. 8(2): p. 165-173.

61. Linster, C.L.,

G. Noel, V. Stroobant,

D. Vertommen, et al., Ethylmalonyl-CoA

Decarboxylase, a New Enzyme Involved in Metabolite Proofreading. Journal of Biological Chemistry, 2011. 286(50): p. 42992-43003.

\section{Acknowledgement}

We acknowledge John and Birthe Meyer Foundation for financial support.

\section{Conflicts of Interest}

The authors have no conflict of interest to declare

\section{Legends:}

Figure 1: A) The levels of CYP450 members in ETHE1 deficient mice compared with healthy controls. The majority of the CYPs members had values below 1.0. B) Proteomics data for redox regulating and oxidative stress response proteins. C) Protein description and fold change for significantly altered proteins involved in glycolysis and gluconeogenesis. D)

22 Significantly altered proteins participating in branched chained amino acid catabolism and

23 fatty acid oxidation. E) Proteomics data for heterogeneous ribonucleoproteins (hnRNPs). F)

24 Class A and class B member of heat shock protein 90 with another heat shock protein 8 were 
1 significantly down-regulated. CDC37 is HSP90 co-chaperone which promotes interactions of

2 kinases and HSP90, resulting in stabilization and promotion of HSP90 activity. HSP90 cochaperone was also down-regulated. G) Structural and cytoskeleton proteins up-regulated in ETHE1-deficient mice liver. Error bars represent \pm SEM. Dark and light grey bars depict significant and non-significant differential protein expression, respectively. Statistical significance levels are illustrated as $\mathrm{p}<0.05$ with $*, \mathrm{p}<0.01$ with $* *, \mathrm{p}<0.001$ with $* * *$.

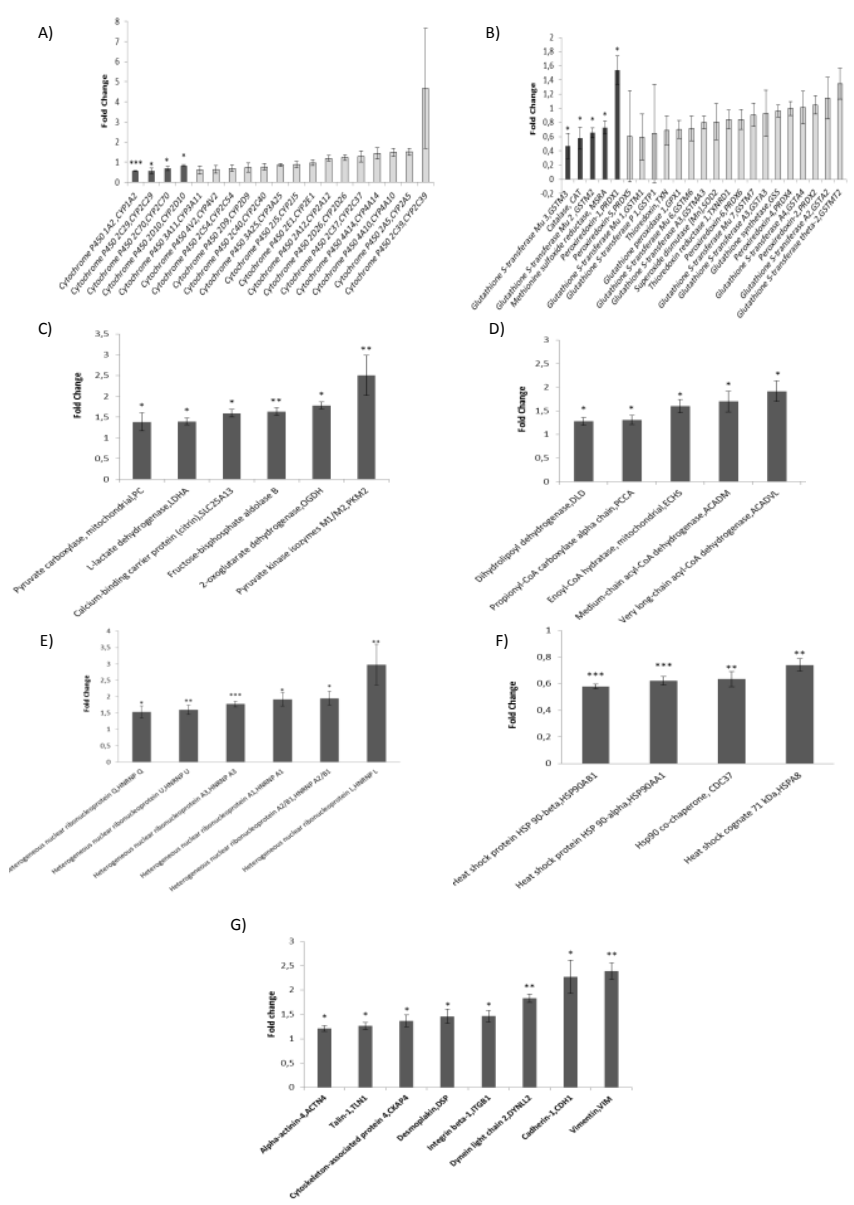

Figure 2: Western blot analysis of acetylated proteins in liver tissue of Ethe1+/+ and Ethe1-/9 mice with anti-acetyl lysine antibody. 


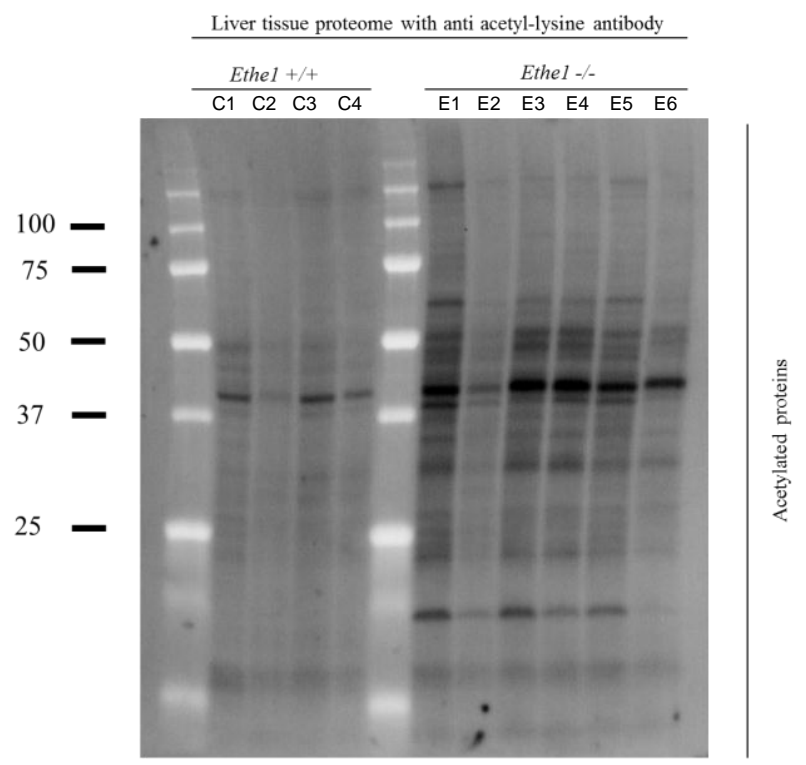

Figure S1. STRING analysis of protein-protein interaction. Protein-protein interaction of 137 significantly altered proteins, A) up-regulated and B) down-regulated were analysed by applying STRING (Search Tool for the Retrieval of Interacting Genes/Proteins) version 10 with the confidence cut off 0.5 . Proteins are represented as nodes with connecting lines. Stronger associations are represented by thicker lines. Disconnected nodes were removed.

Figure S2. The levels of glycolytic enzymes, and lactate dehydrogenase, detected and 8 quantified in the iTRAQ studies. All data are from the $\mathrm{S}$ fraction since all proteins were detected there with higher number of quantitative scans than in the $M$ fraction. Hexokinase and phosphofructokinase escaped detection and are not depicted. Fold change was calculated as the ratio of the mean ETHE1 deficient divided by mean of control mice. Error bars

12 represent \pm SEM. Dark and light grey bars depict significant and non-significant differential 13 protein expression, respectively. Statistical significance levels are illustrated as $p<0.05$ with $*, \mathrm{p}<0.01$ with **, $\mathrm{p}<0.001$ with $* * *$. 
2 Figure S3. SYPRO ruby total protein stain of the Western blot membrane that subsequently was probed for protein acetylation.

Table S1. Identification and quantification data of 1119 protein entries (967 unique proteins) obtained from nLC MS/MS analyses of the mice liver tissue. The data were obtained from raw files from nLC MS/MS subjected to Proteome Discover. Min n denotes the number of peptide scans, and Fraction stands for two phase protein separation ( $M=$ Membrane and S=Supernatant), where 1 and 2 denotes the fraction with highest and lowest number of scans $(\operatorname{Min} \mathrm{N})$, respectively, when the protein was detected in both fractions.

Table S2. 137 significant proteins quantified in Ethe $1^{-/-}$and Ethe $1^{+/+}$mice liver tissue by nLC-MS/MS analysis. Ratio denotes iTRAQ quantitative data from Ethe ${ }^{-/-}$divided by Ethe $1^{+/+}$.

Table 1: Enriched Protein Functional annotation terms (top 5) among the 137 differentially regulated proteins in ETHE1 deficient mice compared with wild type was obtained through analysis in DAVID. Count signifies number of proteins in the cluster. In our study the significant functional annotations were selected using DAVID with the thresholds of enrichment score $>1.3$ and count $>5$.

21

\section{Annotation cluster}

Functional count

P-value

Benjamini 


\begin{tabular}{|c|c|c|c|c|c|}
\hline & annotation & score & & & \\
\hline GOTERM_CC_FAT & $\begin{array}{c}\text { Ribonucleoprotein } \\
\text { complex }\end{array}$ & 2.99 & 9 & $2.9 \mathrm{E}-1$ & $9.6 \mathrm{E}-1$ \\
\hline INTERPRO & RNA recognition motif & 2.99 & 11 & $7.1 \mathrm{E}-6$ & $1.6 \mathrm{E}-3$ \\
\hline GOTERM_BP_FAT & Oxidation Reduction & 2.11 & 20 & $8.0 \mathrm{E}-3$ & $9.7 \mathrm{E}-1$ \\
\hline GOTERM_MF_FAT & $\begin{array}{c}\text { Transition metal ion } \\
\text { binding }\end{array}$ & 2.11 & 15 & $2.9 \mathrm{E}-2$ & $9.2 \mathrm{E}-1$ \\
\hline SP_PIR_KEYWORDS & Metal-binding & 1.68 & 18 & $4.8 \mathrm{E}-3$ & $1.8 \mathrm{E}-1$ \\
\hline GOTERM_CC_FAT & Mitochondrion & 1.3 & 26 & $1.4 \mathrm{E}-3$ & $7.8 \mathrm{E}-2$ \\
\hline
\end{tabular}

Table 2: Proteins of the DAVID cluster "Oxidation -Reduction" and Metal binding proteins not present in the cluster of oxidoreductases. All were down-regulated in ETHE1-deficient mice. Cofactor information was obtained from Uniprot.

\begin{tabular}{|c|c|c|c|c|}
\hline & Protein Name & $\begin{array}{l}\text { Gene } \\
\text { Name }\end{array}$ & $\begin{array}{c}\text { Uniprot } \\
\text { IDs }\end{array}$ & Cofactor \\
\hline \multirow{8}{*}{ 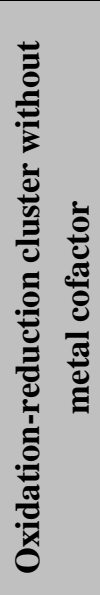 } & 4-trimethylaminobutyraldehyde dehydrogenase & Aldh9a1 & Q9JLJ2 & \\
\hline & cytidine monophospho-N-acetylneuraminic acid hydroxylase & Cmah & Q61419 & \\
\hline & cytosolic 10 -formyltetrahydrofolate dehydrogenase & Aldh111 & Q8R0Y6 & \\
\hline & estradiol 17 beta-dehydrogenase & Akr1c6 & P70694 & \\
\hline & glycine dehydrogenase & Gldc & Q91W43 & \\
\hline & gulonolactone (L-) oxidase & Gulo & P58710 & \\
\hline & methionine sulfoxide reductase A & Msra & Q9D6Y7 & \\
\hline & peroxisomal multifunctional enzyme type 2 & Hsd17b4 & P51660 & \\
\hline
\end{tabular}

This article is protected by copyright. All rights reserved. 


\begin{tabular}{|c|c|c|c|c|}
\hline \multirow{12}{*}{ 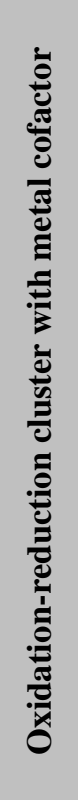 } & 3-Hydroxyanthranilate 3,4-Dioxygenase & Haаo & Q78JT3 & iron \\
\hline & alcohol dehydrogenase 1 (class I) & Adh1 & P00329 & zinc \\
\hline & catalase & Cat & P24270 & iron-sulfur \\
\hline & cytochrome P450, family 1 , subfamily a, polypeptide 2 & Cyp1a2 & P00186 & iron \\
\hline & cytochrome P450, family 2 , subfamily c, polypeptide 29 & Сур2с29 & Q64458 & iron \\
\hline & cytochrome P450, family 2 , subfamily c, polypeptide 70 & Сур2c70 & Q91W64 & iron \\
\hline & cytochrome P450, family 2 , subfamily D, polypeptide 10 & Сур2d10 & Q9EP75 & iron \\
\hline & isocitrate dehydrogenase $1(\mathrm{NADP}+)$, soluble & Idh1 & O88844 & $\begin{array}{l}\text { magnesium or } \\
\text { manganese }\end{array}$ \\
\hline & NADP-dependent malic enzyme & Me1 & P06801 & $\begin{array}{l}\text { magnesium or } \\
\text { manganese }\end{array}$ \\
\hline & ribosyldihydronicotinamide dehydrogenase & $\mathrm{NqO} 2$ & Q9JI75 & zinc \\
\hline & squalene synthase & Fdft1 & P53798 & magnesium \\
\hline & sulfite oxidase & Suox & Q8R086 & $\begin{array}{l}\text { molybdenum- } \\
\text { molybdopterin }\end{array}$ \\
\hline \multirow{6}{*}{ 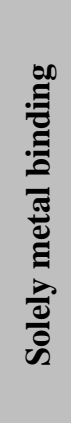 } & cytosol aminopeptidase & Lap3 & Q9CPY7 & zinc \\
\hline & delta-aminolevulinic acid dehydratase & Alad & P10518 & zinc \\
\hline & dipeptidyl peptidase 3 & Dpp3 & Q99KK7 & zinc \\
\hline & fructose bisphosphatase 1 & Fbp1 & Q9QXD6 & magnesium \\
\hline & GTP-binding protein & Sar1b & Q9CQC9 & magnesium \\
\hline & hydroxymethylglutaryl-CoA lyase & Hmgcl & P38060 & $\begin{array}{l}\text { manganese } \\
\text { magnesium }\end{array}$ \\
\hline
\end{tabular}


Table 3: Targeted proteomics data validation by PRM and comparison with iTRAQ proteomics data. VLCAD, HSP90 and CYP1A2 as representing proteins are regulated in same direction in both assays, while ApoE was not significant in PRM data.

\begin{tabular}{|c|c|c|c|c|}
\hline \multicolumn{3}{|c|}{ PRM validation } & \multicolumn{2}{c|}{ iTRAQ proteomics data } \\
\hline Protein & Fold change & $p$ value & Fold change & $p$ value \\
\hline VLCAD & 1.45 & 0.07 & 1.91 & 0.005 \\
\hline HSP90 & 0.66 & 0.0001 & 0.57 & $2.21 \mathrm{E}-05$ \\
\hline CYP1A2 & 0.45 & 0.1 & 0.55 & 0.0003 \\
\hline AP0E & 1.12 & 0.68 & 1.64 & 0.02 \\
\hline ECHD1 & 0.75 & 0.19 & 1.0 & 0.99 \\
\hline ACADS & 1.01 & 0.94 & 1.15 & 0.12 \\
\hline
\end{tabular}

